\title{
Surgical site infection and its associated factors in Ethiopia: a systematic review and meta-analysis
}

\author{
Wondimeneh Shibabaw Shiferaw ${ }^{1 *}$, Yared Asmare Aynalem', Tadesse Yirga Akalu ${ }^{2}$ and \\ Pammla Margaret Petrucka ${ }^{3}$
}

\begin{abstract}
Background: Despite being a preventable complication of surgical procedures, surgical site infections (SSIs) continue to threaten public health with significant impacts on the patients and the health-care human and financial resources. With millions affected globally, there is significant variation in the primary studies on the prevalence of SSIs in Ethiopia. Therefore, this study aimed to estimate the pooled prevalence of SSI and its associated factors among postoperative patients in Ethiopia.
\end{abstract}

Methods: PubMed, Scopus, Psyinfo, African Journals Online, and Google Scholar were searched for studies that looked at SSI in postoperative patients. A funnel plot and Egger's regression test were used to determine publication bias. The $\mathrm{I}^{2}$ statistic was used to check heterogeneity between the studies. DerSimonian and Laird random-effects model was applied to estimate the pooled effect size, odds ratios (ORs), and 95\% confidence interval (Cls) across studies. The subgroup analysis was conducted by region, sample size, and year of publication. Sensitivity analysis was deployed to determine the effect of a single study on the overall estimation. Analysis was done using STATA ${ }^{\text {TM }}$ Version 14 software.

Result: A total of 24 studies with 13,136 study participants were included in this study. The estimated pooled prevalence of SSI in Ethiopia was 12.3\% (95\% Cl: 10.19, 14.42). Duration of surgery > $1 \mathrm{~h}$ (AOR $=1.78 ; 95 \% \mathrm{Cl}: 1.08-$ 2.94), diabetes mellitus (AOR $=3.25 ; 95 \% \mathrm{Cl}: 1.51-6.99)$, American Society of Anaesthesiologists score $>1$ ( $\mathrm{AOR}=2.51$; $95 \% \mathrm{Cl}: 1.07-5.91)$, previous surgery (AOR $=2.5 ; 95 \% \mathrm{Cl}: 1.77-3.53)$, clean-contaminated wound (AOR $=2.15 ; 95 \% \mathrm{Cl}$ : 1.52-3.04), and preoperative hospital stay $>7$ day ( $\mathrm{AOR}=5.76 ; 95 \% \mathrm{Cl}$ : 1.15-28.86), were significantly associated with SSI.

Conclusion: The prevalence of SSI among postoperative patients in Ethiopia remains high with a pooled prevalence of $12.3 \%$ in 24 extracted studies. Therefore, situation based interventions and region context-specific preventive strategies should be developed to reduce the prevalence of SSI among postoperative patients.

Keywords: Surgical site infections, Wound infection, Postoperative, Ethiopia

\footnotetext{
* Correspondence: wshibabaw2015@gmail.com

'Department of Nursing, College of Health Science, Debre Berhan University, P.O. Box 445, Debre Berhan, Ethiopia

Full list of author information is available at the end of the article
}

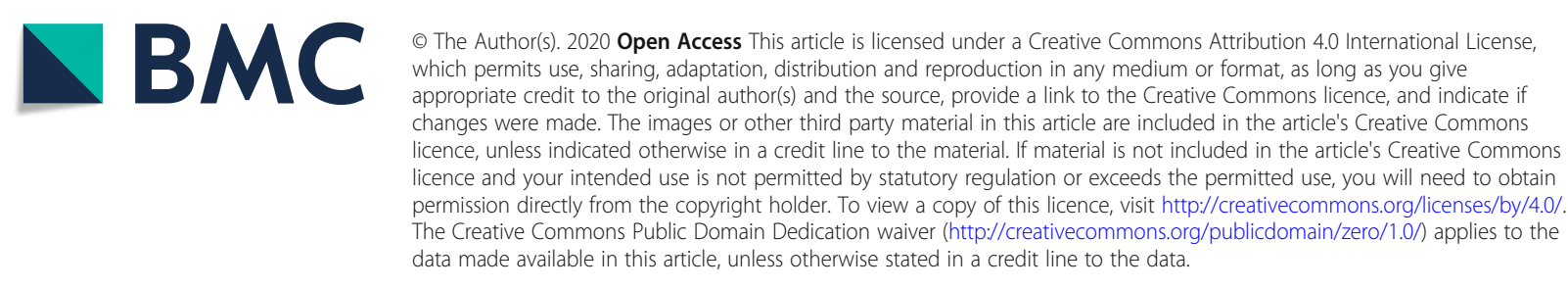




\section{Background}

Surgical site infections (SSIs) are infections that occur at or near surgical incision within 30 days of operation or after 1 year if an implant is placed [1]. SSIs are a major cause of morbidity and mortality worldwide, affecting $5.6 \%$ of surgical procedures in developing countries [2]. According to a World Health Organization (WHO) report, the incidence of SSIs ranges from 1.2 to 23.6 per 100 surgical procedures [3]. Worldwide, it has been reported that more than one-third of postoperative deaths are related to SSIs [4]. In addition, SSIs threaten the lives of millions of patients each year and contribute to the spread of antibiotic resistance [5].

The incidence of SSIs is higher in developing countries relative to developed nations [6], reported as the second most common cause of hospital acquired infection (HAI) in Europe and the United States of America (USA) [7]. Approximately $2-5 \%$ of surgical patients worldwide have developed SSIs [8]. SSIs are the most frequent type of HAI in low and middle income countries (LMICs) and affect up to one third of patients who have undergone a surgical procedure $[9,10]$. In LMICs, the pooled incidence of SSI was 11.8 per 100 surgical procedures [11]. In Africa, SSIs were the leading infections in hospitals and incidence ranged from $2.5-30.9 \%$ [12]. Substantial evidentiary variation on the prevalence of SSI exists across the globe, such as $10.56 \%$ in Nnewi [13], 53\% in Iran [14], and 16.4\% in Uganda [15].

According to recent evidence, the risk factors for SSI are multifactorial and complex. For instance, preexisting illness [16-18], wound contamination [13, 15, 19], American Society of Anaesthesiologists (ASA) score III or IV [13, 20], non-use of prophylactic antibiotics [21], presence of hypovolemic [22], longer duration of operation $[16,17,21]$, longer preoperative hospital stay $[16,21]$, postoperative hospital stay $[16,19]$, advanced age $[16,21]$, alcohol use $[19,23]$, previous surgery [23], use of drain [17], use of iodine alone in skin preparation [17], smoking [17, 18], absence of wound care [18], and hair removal inside operating room [20] were factors associated with SSIs.

Though SSIs are among the most preventable healthcare-associated infections. However, according to the available global evidence, SSIs impose significant burden to the patient and health care system in terms of prolonged hospital stays [24], spend time in an intensive care unit [7], readmission to hospital [25], long-term disability [26], contribute to spread of antibiotic resistance [3, 11], increase treatment intensity [24], substantial financial burden to health care systems $[7,26]$, high costs for patients and families $[7,27,28]$, deterioration in the quality of life [29], and unnecessary deaths [3, 7, 26]. Effectively controlling SSIs can reduce some of these negative effects [11], as up to one-half of SSIs can generally be prevented through an improved adherence to established basic principles, such as surgical hand preparation, skin antisepsis, adequate antibiotic prophylaxis, less traumatic, less invasive and shorter surgery duration, improved haemostasis and avoidance of hypothermia [7, 23, 30].

Despite improvements in operating room practices, instrument sterilization methods, better surgical technique, and the best efforts of infection prevention strategies, surgical site infections remain a major cause of hospital acquired infections. The need for SSIs prevention policies has been recognized in Ethiopia. Different primary studies in Ethiopia show the magnitude of SSIs as a health issue in the region; however, incidence rates are inconclusive. Therefore, this review and metaanalysis aimed to estimate the pooled national prevalence of SSI and its associated factors in Ethiopia.

\section{Methods \\ Literature search strategy}

Initially, Cochrane library, JBI and PROSPERO databases were searched. To confirm whether systematic review and meta-analysis is exist or for the presence of ongoing projects related to the current topic. The literature was searched using PubMed, Scopus, Google scholar, African journals online, and PsyINFO. Relevant articles were identified according to the following terms: "surgical site infections", "surgical incision infection", "postoperative", "wound infection", "predictor", "associated factors", "Ethiopia". The key terms were used in combination using Boolean operators like "OR" or "AND". The searches were restricted to full texts, free articles, human studies, and English language publications. This search involved articles published from January 1st, 2000 to November 11th, 2019. Grey literatures, such as surveillance reports, academic dissertations, conference abstracts, were examined. In addition the reference lists of included articles were hand-searched to identify any potential additional relevant articles.

\section{Eligibility criteria}

Studies were included in the meta-analysis if they adhered to the following guidelines: (1) all observational study designs (cross-sectional, case-control, and cohort studies) needed to report the prevalence of SSI; (2 published from 2000 to 2019; (3) published in English language; (4) abstract and full text were available for this review; and (5) conducted in Ethiopia. Studies were excluded if they: (1) possessed a poor quality score as per the stated criteria; (2) failed to determine the desired outcome (SSI); (3) were not fully accessible; and (4) included only in cesarean section patients. 


\section{Outcome of interest}

The main outcome of interest was the prevalence of SSIs reported in the original paper both as percentage or as the number of SSIs cases(n) / total number of patients who undergo surgery $(\mathrm{N})$. These two parameters were necessary to calculate the pooled prevalence of SSIs in the meta-analysis. Therefore, the prevalence was calculated by dividing the number of individuals who have SSIs to the total number of patients who undergo surgery (sample size) multiplied by 100 .

\section{Data extraction}

Two authors independently extracted all necessary data from each study using a standardized data extraction format. If discrepancies between data extractors were observed, a third author was involved. For each included study, the following data were extracted: primary author, publication year, region, study design, sample size, prevalence of SSIs, and associated factors (wound type, preoperative hospital stay, duration of operation, history surgery, ASA score, diabetes mellitus, smoking, preoperative blood transfusion). We also contacted corresponding authors (by e-mail) of articles that did not provide details of their study's background and asked them for the relevant information, such as study time, region, or hospital.

\section{Quality assessment}

Two independent investigators assessed the methodological quality of all of the potential studies to be included in our analysis. Any disagreements between the authors were resolved through discussion or, if consensus could not be reached, consultation with a third, independent author was undertaken. The quality of each included study was assessed using the Joanna Briggs Institute (JBI) quality appraisal checklist [31]. This scale has several key criteria to appraise cohort studies including: [1] similarity of groups, [2] similarity of exposure measurement, [3] validity and reliability of measurement, [4] identification of confounder, [5] strategies to deal with confounder, [6] appropriateness of groups/participants at the start of the study, [7] validity and reliability of outcome measured, [8] sufficiency of follow-up time, [9] completeness of follow-up or descriptions of reason to loss to follow-up, [10] strategies to address incomplete follow-up, and [11] appropriateness of statistical analysis. The items used to appraise cross-sectional studies were: [1] inclusion criteria, [2] description of study subject and setting, [3] valid and reliable measurement of exposure, [4] objective and standard criteria used, [5] identification of confounder, [6] strategies to handle confounder, [7] outcome measurement, and [8] appropriate statistical analysis. Studies were considered low risk when scored at $50 \%$ and above on the quality assessment indicators.

\section{Statistical analysis}

To obtain the pooled prevalence of surgical site infection, a meta-analysis using random-effects DerSimonian and Laird model was performed due to anticipated heterogeneity [32]. Cochran's Q chi-square statistics and $\mathrm{I}^{2}$ statistical test was conducted to assess the random variations between primary studies [33]. In this study, heterogeneity was interpreted as an $\mathrm{I}^{2}$ value of $0 \%=$ no heterogeneity, $25 \%=$ low, $50 \%=$ moderate, and $75 \%=$ high [34]. In case of high heterogeneity, subgroup analysis and sensitivity analyses were run to identify possible moderators of this heterogeneity. Potential publication bias was assessed by visually inspecting funnel plots and objectively using the Egger bias test [35]. To account for any publication bias, we used the trim-and-fill method, based on the assumption that the effect sizes of all the studies are normally distributed around the center of a funnel plot. The meta-analysis was performed using the STATA $^{\mathrm{Tu}}$ Version 14 software [36]. Finally, for all analyses, $P<0.05$ was considered statistically significant.

\section{Presentation and reporting of results}

The results of this review were reported based on the Preferred Reporting Items for Systematic Review and Meta-Analysis statement (PRISMA) guideline [37], and PRISMA checklist has been used. The entire process of study screening, selection, and inclusion were shown with the support of a flow diagram. Additionally, tables and narrative summaries were used to report the risk of bias for every eligible study.

\section{Results}

\section{Search results}

The search strategy identified a total of 293 articles. Of these, 289 articles were retrieved from PubMed (45), Scopus (31), Google Scholar (152), Psyinfo (29) and African Journals Online (59). The remaining 4 were found through a manual search. After excluding duplicate publications, 168 articles remained. About 113 articles were excluded after reading the titles and abstracts based on the pre-defined eligibility criteria. Out of them 55 articles were included and screened for further assessment. Finally, 24 articles included in the analysis. The detailed steps of the screening process are shown in a PRISMA flow chart of the study selection (Fig. 1).

\section{Baseline characteristics of included studies}

A total of 24 studies with 13,136 study participants were included in this systematic review and meta-analysis. Regarding study design, two thirds $(62.5 \%)$ of the studies included were cross-sectional. The number of 


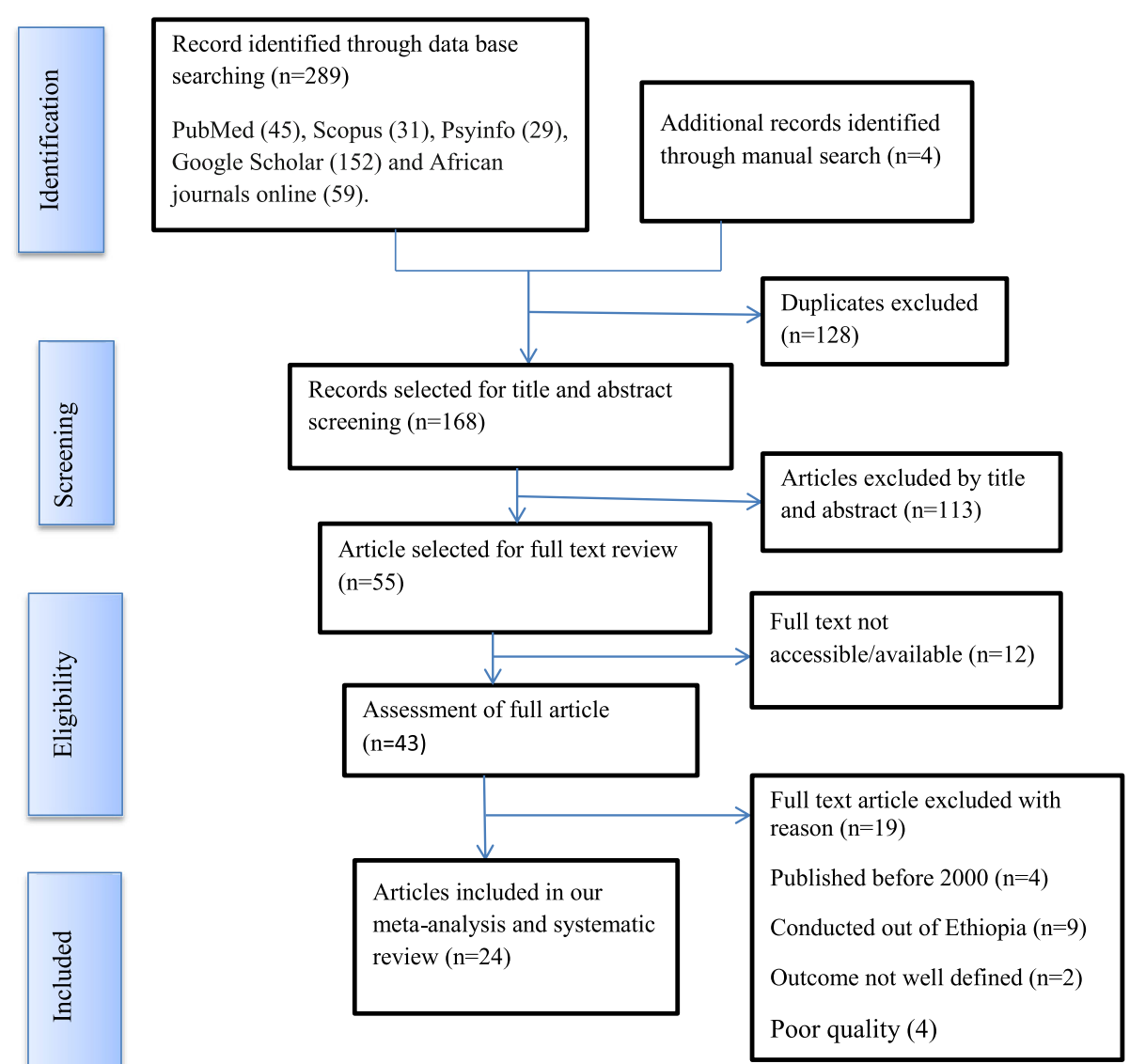

Fig. 1 PIRSMA Flowchart diagram of the study selection

participants per study ranged from 65 to 1754 . The highest prevalence (36\%) of SSIs was reported in a study conducted in Oromia [38]. Whereas the lowest prevalence $(3.5 \%)$ was reported in a study conducted in Amhara [39]. The prevalence of SSIs in post-operative patients was obtained from various regions in Ethiopia. Seven of the studies included in this review were from Addis Ababa [23, 40-45], eight from Amhara [16, 18, $39,46-50]$, four from Oromia [38, 51-53], three from Tigray $[19,54,55]$, and two from SNNPR $[21,56]$. With respect to sampling technique, 12 studies [16, 21, 38, 39, $42,47,49-52,54,56]$ used the consecutive sampling technique to select study participants. But, two studies $[44,55]$ were not specified the sampling methods they used. The quality score of each primary study, based on the JBI quality appraisal criteria, showed no considerable risk; hence, all the studies were considered in this review (Table 1).

\section{Prevalence of surgical site infection}

The current meta-analysis using the random effects model showed that the estimated overall prevalence of SSI in post-operative patients in Ethiopia was $12.3 \%$
(95\% CI: $10.19,14.42)$ with a significant level of heterogeneity $\left(\mathrm{I}^{2}=93.8 \% ; p<0.001\right)$ (Fig. 2 ).

\section{Subgroup analysis}

The presence of significant heterogeneity among the primary studies requires the need to conduct subgroup analysis. In order to identify the sources of heterogeneity, we deployed sub-group analysis using publication year, sample size, region, study design, and sampling technique to determine the pooled prevalence of SSIs (Table 2). The prevalence of SSIs was found to be $17.19 \%$ in SSNPR, $13.08 \%$ in studies published since 2010, $14.18 \%$ in studies with cohort design, $15.54 \%$ a sample size less than or equal to 300 , and $13.38 \%$ in studies with probability sampling technique of our included primary studies.

\section{Publication bias}

In the present review there was publication bias among the included studies, as illustrated by the asymmetrical distribution of our funnel plot (Fig. 3). Likewise, the result of Egger's test was statistically significant for the presence of publication bias $(P=0.001)$. In addition, to 
Table 1 Baseline characteristics of the included studies

\begin{tabular}{|c|c|c|c|c|c|c|c|c|c|c|}
\hline Author & $\begin{array}{l}\text { Publication } \\
\text { year }\end{array}$ & Region & $\begin{array}{l}\text { Study } \\
\text { design }\end{array}$ & $\begin{array}{l}\text { Sample } \\
\text { size }\end{array}$ & $\begin{array}{l}\text { Prevalence } \\
(\%)\end{array}$ & $\begin{array}{l}\text { Data collection } \\
\text { method }\end{array}$ & $\begin{array}{l}\text { Sampling } \\
\text { technique }\end{array}$ & $\begin{array}{l}\text { Types of } \\
\text { surgery }\end{array}$ & Study period & $\begin{array}{l}\text { Quality } \\
\text { Score }\end{array}$ \\
\hline $\begin{array}{l}\text { Afenigus.A.et } \\
\text { al [18] }\end{array}$ & 2019 & Amhara & $\begin{array}{l}\text { Cross- } \\
\text { sectional }\end{array}$ & 165 & 25.5 & $\begin{array}{l}\text { Interview and clinical } \\
\text { sign }\end{array}$ & Systematic & $\begin{array}{l}\text { General } \\
\text { Surgery }\end{array}$ & $\begin{array}{l}\text { March to May, } \\
2017\end{array}$ & $\begin{array}{l}\text { Low } \\
\text { risk }\end{array}$ \\
\hline $\begin{array}{l}\text { Alamrew K } \\
\text { [40]. }\end{array}$ & 2018 & $\begin{array}{l}\text { Addis } \\
\text { Ababa }\end{array}$ & $\begin{array}{l}\text { Cross- } \\
\text { sectional }\end{array}$ & 413 & 11.1 & Chart review & $\begin{array}{l}\text { Simple } \\
\text { random }\end{array}$ & $\begin{array}{l}\text { General } \\
\text { Surgery }\end{array}$ & $\begin{array}{l}\text { June } 10 \text { to } \\
\text { September } 10,2016\end{array}$ & $\begin{array}{l}\text { Low } \\
\text { risk }\end{array}$ \\
\hline Ali S.et al. [51] & 2018 & Oromia & Cohort & 1069 & 13.85 & $\begin{array}{l}\text { Laboratory and } \\
\text { interview }\end{array}$ & Consecutive & $\begin{array}{l}\text { General } \\
\text { Surgery }\end{array}$ & $\begin{array}{l}\text { May to September, } \\
2016\end{array}$ & $\begin{array}{l}\text { Low } \\
\text { risk }\end{array}$ \\
\hline Amare B [39] & 2011 & Amhara & $\begin{array}{l}\text { Cross- } \\
\text { sectional }\end{array}$ & 1627 & 3.5 & Clinical sign & Consecutive & $\begin{array}{l}\text { General } \\
\text { Surgery }\end{array}$ & $\begin{array}{l}\text { January } 2010 \text { to } \\
\text { June } 2010\end{array}$ & $\begin{array}{l}\text { Low } \\
\text { risk }\end{array}$ \\
\hline $\begin{array}{l}\text { Amenu D. } \\
\text { et al. [52] }\end{array}$ & 2011 & Oromia & $\begin{array}{l}\text { Cross- } \\
\text { sectional }\end{array}$ & 770 & 11.4 & Interview, logbook & Consecutive & $\begin{array}{l}\text { Obstetric } \\
\text { Surgery }\end{array}$ & $\begin{array}{l}\text { April 1, } 2009 \text { to } \\
\text { March } 312,010\end{array}$ & $\begin{array}{l}\text { Low } \\
\text { risk }\end{array}$ \\
\hline $\begin{array}{l}\text { Argaw NA. } \\
\text { et al. [41] }\end{array}$ & 2017 & $\begin{array}{l}\text { Addis } \\
\text { Ababa }\end{array}$ & $\begin{array}{l}\text { Cross- } \\
\text { sectional }\end{array}$ & 200 & 16 & Chart review & All included & $\begin{array}{l}\text { General } \\
\text { Surgery }\end{array}$ & NR & $\begin{array}{l}\text { Low } \\
\text { risk }\end{array}$ \\
\hline $\begin{array}{l}\text { Billoro BB. } \\
\text { et al. [56] }\end{array}$ & 2019 & SNNPR & cohort & 280 & 16.5 & $\begin{array}{l}\text { Interview, chart } \\
\text { review, laboratory }\end{array}$ & Consecutive & $\begin{array}{l}\text { General } \\
\text { Surgery }\end{array}$ & $\begin{array}{l}\text { January } 1 \text { to } \\
\text { September 1, } 2017\end{array}$ & $\begin{array}{l}\text { Low } \\
\text { risk }\end{array}$ \\
\hline $\begin{array}{l}\text { Endalafer N } \\
\text { [42]. }\end{array}$ & 2011 & $\begin{array}{l}\text { Addis } \\
\text { Ababa }\end{array}$ & $\begin{array}{l}\text { Cross- } \\
\text { sectional }\end{array}$ & 215 & 17.67 & Laboratory & Consecutive & $\begin{array}{l}\text { General } \\
\text { Surgery }\end{array}$ & $\begin{array}{l}\text { June } 2007 \text { to April } \\
2008\end{array}$ & $\begin{array}{l}\text { Low } \\
\text { risk }\end{array}$ \\
\hline $\begin{array}{l}\text { Fisha K. et al. } \\
\text { [46] }\end{array}$ & 2019 & Amhara & Cohort & 642 & 9.9 & Chart review & $\begin{array}{l}\text { Simple } \\
\text { random }\end{array}$ & $\begin{array}{l}\text { General } \\
\text { Surgery }\end{array}$ & $\begin{array}{l}\text { March } 15 \text { to April } \\
15,2018\end{array}$ & $\begin{array}{l}\text { Low } \\
\text { risk }\end{array}$ \\
\hline $\begin{array}{l}\text { Forrester JA. } \\
\text { et al. [38] }\end{array}$ & 2018 & Oromia & Cohort & 65 & 36 & Clinical sign & Consecutive & $\begin{array}{l}\text { Obstetric } \\
\text { Surgery }\end{array}$ & $\begin{array}{l}\text { September } 9 \text { to } 20 \text {, } \\
2016\end{array}$ & $\begin{array}{l}\text { Low } \\
\text { risk }\end{array}$ \\
\hline $\begin{array}{l}\text { Gebremeskel } \\
\text { S [43]. }\end{array}$ & 2018 & $\begin{array}{l}\text { Addis } \\
\text { Ababa }\end{array}$ & $\begin{array}{l}\text { Cross- } \\
\text { sectional }\end{array}$ & 410 & 4.8 & $\begin{array}{l}\text { Chart review, } \\
\text { interview and } \\
\text { laboratory }\end{array}$ & Systematic & $\begin{array}{l}\text { General } \\
\text { Surgery }\end{array}$ & $\begin{array}{l}\text { March } 1 \text { to August } \\
30,2017\end{array}$ & $\begin{array}{l}\text { Low } \\
\text { risk }\end{array}$ \\
\hline $\begin{array}{l}\text { Gedefaw.G. } \\
\text { et al. [47] }\end{array}$ & 2018 & Amhara & $\begin{array}{l}\text { Cross- } \\
\text { sectional }\end{array}$ & 447 & 9.6 & Chart review & Consecutive & $\begin{array}{l}\text { Obstetric } \\
\text { Surgery }\end{array}$ & $\begin{array}{l}\text { March } 1 \text { to April } \\
30,2018\end{array}$ & $\begin{array}{l}\text { Low } \\
\text { risk }\end{array}$ \\
\hline $\begin{array}{l}\text { Gelaw A. et al. } \\
\text { [48] }\end{array}$ & 2014 & Amhara & $\begin{array}{l}\text { Cross- } \\
\text { sectional }\end{array}$ & 510 & 8.2 & Laboratory & All included & $\begin{array}{l}\text { General } \\
\text { Surgery }\end{array}$ & $\begin{array}{l}\text { November } 2010 \text { to } \\
\text { February } 2011\end{array}$ & $\begin{array}{l}\text { Low } \\
\text { risk }\end{array}$ \\
\hline $\begin{array}{l}\text { Halawi.E. et al. } \\
\text { [23] }\end{array}$ & 2018 & $\begin{array}{l}\text { Addis } \\
\text { Ababa }\end{array}$ & Cohort & 158 & 20.6 & $\begin{array}{l}\text { Clinical sign, chart } \\
\text { review, laboratory }\end{array}$ & Convenient & $\begin{array}{l}\text { General } \\
\text { Surgery }\end{array}$ & $\begin{array}{l}\text { April } 1 \text { to April 30, } \\
2017\end{array}$ & $\begin{array}{l}\text { Low } \\
\text { risk }\end{array}$ \\
\hline $\begin{array}{l}\text { Laloto TL. } \\
\text { et al. [21] }\end{array}$ & 2017 & SNNPR & Cohort & 127 & 19.1 & $\begin{array}{l}\text { Chart review, clinical } \\
\text { sign, interview }\end{array}$ & Consecutive & $\begin{array}{l}\text { General } \\
\text { Surgery }\end{array}$ & $\begin{array}{l}\text { March } 2 \text { to May 2, } \\
2015\end{array}$ & $\begin{array}{l}\text { Low } \\
\text { risk }\end{array}$ \\
\hline $\begin{array}{l}\text { Mamo T.et al. } \\
{[53]}\end{array}$ & 2017 & Oromia & Cohort & 390 & 9.4 & $\begin{array}{l}\text { Laboratory, chart } \\
\text { review, interview }\end{array}$ & Purposive & $\begin{array}{l}\text { Obstetric } \\
\text { Surgery }\end{array}$ & $\begin{array}{l}\text { April } 23 \text { to } \\
\text { September 5, } 2015\end{array}$ & $\begin{array}{l}\text { Low } \\
\text { risk }\end{array}$ \\
\hline $\begin{array}{l}\text { Melku S.et al. } \\
\text { [49] }\end{array}$ & 2012 & Amhara & $\begin{array}{l}\text { Cross- } \\
\text { sectional }\end{array}$ & 1383 & 8 & $\begin{array}{l}\text { Interview, chart } \\
\text { review, laboratory }\end{array}$ & Consecutive & $\begin{array}{l}\text { General } \\
\text { Surgery }\end{array}$ & $\begin{array}{l}\text { April to August } \\
2009\end{array}$ & $\begin{array}{l}\text { Low } \\
\text { risk }\end{array}$ \\
\hline $\begin{array}{l}\text { Mengesha } \\
\text { RE.et al. [54] }\end{array}$ & 2014 & Tigray & $\begin{array}{l}\text { Cross- } \\
\text { sectional }\end{array}$ & 610 & 20.9 & $\begin{array}{l}\text { Clinical sign and } \\
\text { chart review }\end{array}$ & Consecutive & $\begin{array}{l}\text { General } \\
\text { Surgery }\end{array}$ & $\begin{array}{l}\text { January to June } \\
2012\end{array}$ & $\begin{array}{l}\text { Low } \\
\text { risk }\end{array}$ \\
\hline $\begin{array}{l}\text { Mulu W. et al. } \\
{[16]}\end{array}$ & 2013 & Amhara & $\begin{array}{l}\text { Cross- } \\
\text { sectional }\end{array}$ & 294 & 10.2 & $\begin{array}{l}\text { Clinical sign, culture, } \\
\text { and chart review }\end{array}$ & Consecutive & $\begin{array}{l}\text { General } \\
\text { Surgery }\end{array}$ & $\begin{array}{l}\text { October } 2010 \text { to } \\
\text { January } 2011\end{array}$ & $\begin{array}{l}\text { Low } \\
\text { risk }\end{array}$ \\
\hline Taye M [44]. & 2005 & $\begin{array}{l}\text { Addis } \\
\text { Ababa }\end{array}$ & Cohort & 1754 & 14.8 & $\begin{array}{l}\text { Clinical sign and } \\
\text { laboratory }\end{array}$ & Consecutive & $\begin{array}{l}\text { General } \\
\text { Surgery }\end{array}$ & $\begin{array}{l}\text { January } 1,1999 \text { to } \\
\text { Dec 31, } 1999\end{array}$ & $\begin{array}{l}\text { Low } \\
\text { risk }\end{array}$ \\
\hline Tekie K [45]. & 2008 & $\begin{array}{l}\text { Addis } \\
\text { Ababa }\end{array}$ & Cohort & 173 & 17.9 & $\begin{array}{l}\text { Clinical sign and } \\
\text { laboratory }\end{array}$ & All included & $\begin{array}{l}\text { General } \\
\text { Surgery }\end{array}$ & April to July 2006 & $\begin{array}{l}\text { Low } \\
\text { risk }\end{array}$ \\
\hline $\begin{array}{l}\text { Tesfahunegn } \\
\text { Z. et al. [55] }\end{array}$ & 2009 & Tigray & $\begin{array}{l}\text { Cross- } \\
\text { sectional }\end{array}$ & 246 & 7.3 & $\begin{array}{l}\text { Chart review and } \\
\text { clinical sign }\end{array}$ & NR & $\begin{array}{l}\text { General } \\
\text { Surgery }\end{array}$ & $\begin{array}{l}\text { November } 2005 \text { to } \\
\text { April } 2006\end{array}$ & $\begin{array}{l}\text { Low } \\
\text { risk }\end{array}$ \\
\hline $\begin{array}{l}\text { Weldu MG. } \\
\text { et al. [19] }\end{array}$ & 2018 & Tigray & $\begin{array}{l}\text { Cross- } \\
\text { sectional }\end{array}$ & 280 & 11.1 & $\begin{array}{l}\text { Interviewer, clinical } \\
\text { sign }\end{array}$ & $\begin{array}{l}\text { Simple } \\
\text { random }\end{array}$ & $\begin{array}{l}\text { General } \\
\text { Surgery }\end{array}$ & $\begin{array}{l}\text { February } 2 \text { to } \\
\text { March 31, } 2016\end{array}$ & $\begin{array}{l}\text { Low } \\
\text { risk }\end{array}$ \\
\hline $\begin{array}{l}\text { Yallew WW. Et } \\
\text { al [50] }\end{array}$ & 2016 & Amhara & $\begin{array}{l}\text { Cross- } \\
\text { sectional }\end{array}$ & 908 & 7.6 & $\begin{array}{l}\text { Clinical sign, } \\
\text { laboratory, chart } \\
\text { review }\end{array}$ & Consecutive & $\begin{array}{l}\text { General } \\
\text { Surgery }\end{array}$ & March to July 2015 & $\begin{array}{l}\text { Low } \\
\text { risk }\end{array}$ \\
\hline
\end{tabular}




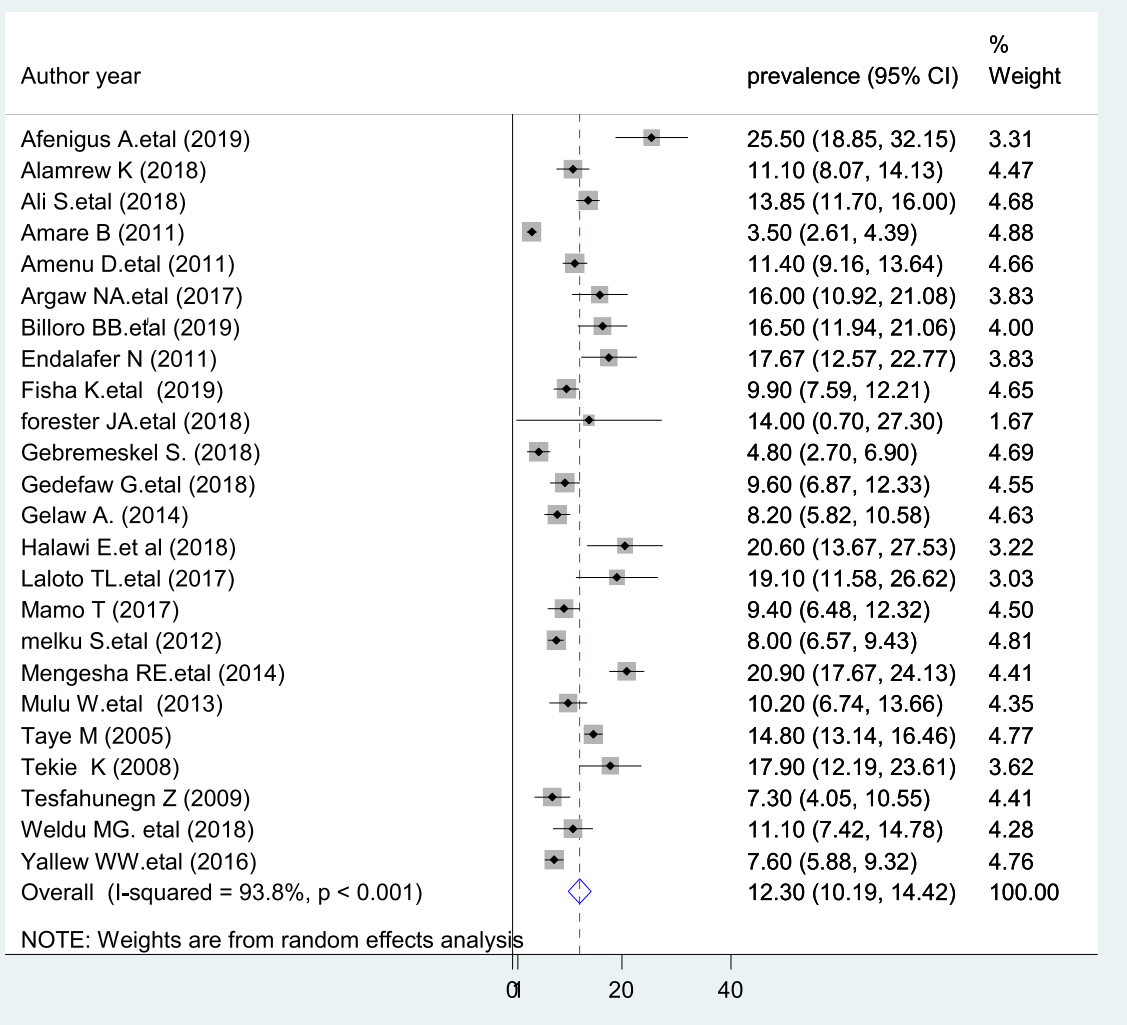

Fig. 2 Forest plot of the prevalence of surgical site infections in post-operative patients

reduce and adjust publication bias, trim and fill analysis was also performed (Fig. 4). Trim and fill analysis is a nonparametric method for estimating the number of missing studies that might exist.

\section{Sensitivity analysis}

Sensitivity analyses using the random effects model revealed that no single study influenced the overall prevalence of SSIs in post-operative patients (Fig. 5).

\section{Associated factors}

Based on this meta-analysis, SSIs in the Ethiopian context were associated with duration of operation, smoking, preoperative blood transfusion, diabetes mellitus, ASA score, previous surgery, wound type, and preoperative hospital stay.

\section{Duration of operation}

Based on the pooled effects of six studies [16, 21, 23, 40, $47,56]$, duration of operation greater than one hour were nearly two times more likely to develop SSI as compared with patients whose operation was completed within one hour (AOR, 1.78, 95\% CI 1.08, 2.94, $\mathrm{I}^{2}=$
54.2\%) (Fig. 6). The evidence from Egger's regression test shows that there was no publication bias $(P=0.803)$.

\section{Preoperative blood transfusion}

According to our current meta-analysis, for those clients who had preoperative blood transfusion were 2.51 times more likely to develop SSIs as compared with patients with no preoperative blood transfusion (AOR, 2.51, 95\% CI $0.67,9.45, \mathrm{I}^{2}=62 \%$ ), although this association was not statistically significant (Fig. 7). The result of the Egger's regression test showed no evidence of publication bias $(P=0.838)$.

\section{Cigarette smoking}

Those patients who smoke cigarettes were 95\% more likely to develop SSI compared with patients who do not smoke cigarettes [OR $=1.95 ; 95 \% \mathrm{CI}: 0.27,13.18, \mathrm{I}^{2}=$ $0.0 \%]$, though not statistically significant (Fig. 8). The evidence from Egger's regression test showed that there was no publication bias $(P=0.107)$.

\section{Diabetes mellitus}

The pooled effects of five studies [16, 18, 21, 47, 53] indicated that those patients who had diabetes mellitus at 
Table $\mathbf{2}$ The results of subgroup analysis by characteristics of the studies

\begin{tabular}{|c|c|c|c|c|}
\hline Subgroup & No. of studies & Prevalence $(95 \% \mathrm{Cl})$ & $\mathrm{I}^{2}(\%)$ & $p$-value \\
\hline \multicolumn{5}{|l|}{ Publication year } \\
\hline Before 2010 & 3 & $13.08(7.37,18.78)$ & $89.2 \%$ & $<0.001$ \\
\hline After 2010 & 21 & $12.60(10.34,14.86)$ & $93.7 \%$ & $<0.001$ \\
\hline \multicolumn{5}{|l|}{ Region } \\
\hline Amhara & 8 & $9.36(6.71,12.00)$ & $93 \%$ & $<0.001$ \\
\hline Oromia & 4 & $11.78(9.56,13.99)$ & $52 \%$ & $<0.001$ \\
\hline Tigray & 3 & $13.11(4.86,21.36)$ & $94.4 \%$ & $<0.001$ \\
\hline Addis Ababa & 7 & $14.26(9.87,18.65)$ & $91.8 \%$ & $<0.001$ \\
\hline SNNPR & 2 & $17.19(13.30,21.09)$ & $0.0 \%$ & 0.562 \\
\hline \multicolumn{5}{|l|}{ Study design } \\
\hline Cross-sectional & 15 & $11.0(8.56,13.43)$ & $93.9 \%$ & $<0.001$ \\
\hline Cohort & 9 & $14.18(11.88,16.48)$ & $72.8 \%$ & $<0.001$ \\
\hline \multicolumn{5}{|l|}{ Sample size } \\
\hline$\leq 300$ & 11 & $15.54(12.26,18.82)$ & $77.9 \%$ & $<0.001$ \\
\hline$>300$ & 13 & $10.15(7.54,12.93)$ & $95.7 \%$ & $<0.001$ \\
\hline \multicolumn{5}{|l|}{ Sampling technique } \\
\hline Probability & 5 & $13.38(8.983,17.78)$ & $84.2 \%$ & $<0.001$ \\
\hline Non probability & 17 & $12.16(9.20,15.12)$ & $94.9 \%$ & $<0.001$ \\
\hline Not specified & 2 & $11.88(7.90,15.87)$ & $92.4 \%$ & $<0.001$ \\
\hline \multicolumn{5}{|l|}{ Type of surgery } \\
\hline General surgery & 20 & $12.68(10.24,15.11)$ & $94.8 \%$ & $<0.001$ \\
\hline Obstetric surgery & 4 & $10.39(8.91,11.81)$ & $0.0 \%$ & 0.61 \\
\hline
\end{tabular}

the time of surgery were 3.25 time more likely to develop SSIs than non-diabetic patients $[\mathrm{AOR}=3.25$; $95 \%$ CI: $1.51,6.99, \mathrm{I}^{2}=21.6 \%$ ] (Fig. 9). The evidence from Egger's test shows no significant proof of publication bias $(P=0.429)$.

\section{ASA score}

The present review revealed that patients with ASA score $>1$ were 2.51 times more likely to develop SSIs compared with ASA score $\leq 1$ [AOR $=2.51 ; 95 \% \mathrm{CI}$ : 1.07 , 5.91, $\mathrm{I}^{2}=0 \%$ ] (Fig. 10). The evidence from Egger's test shows no significant proof of publication bias $(P=$ 0.427).

\section{Previous surgery}

Patients with a history of previous surgery were 2.5 times more likely to develop SSIs compared with patients having no history of previous surgery $[\mathrm{AOR}=2.5$; 95\% CI: 1.77, 3.53, $\mathrm{I}^{2}=0 \%$ ] (Fig. 11). The result of the Egger's regression test showed no evidence of publication bias $(P=0.071)$.

\section{Wound type}

The pooled effects of six studies [21, 23, 40, 41, 51, 56] showed that those patients who have cleancontaminated wound were 2.15 time more likely to develop SSIs than those who have clean wound [AOR $=2.15 ; 95 \%$ CI: 1.52, 3.04, $\mathrm{I}^{2}=0 \%$ ] (Fig. 12). The evidence from Egger's regression test showed that there was no publication bias $(P=0.15)$.

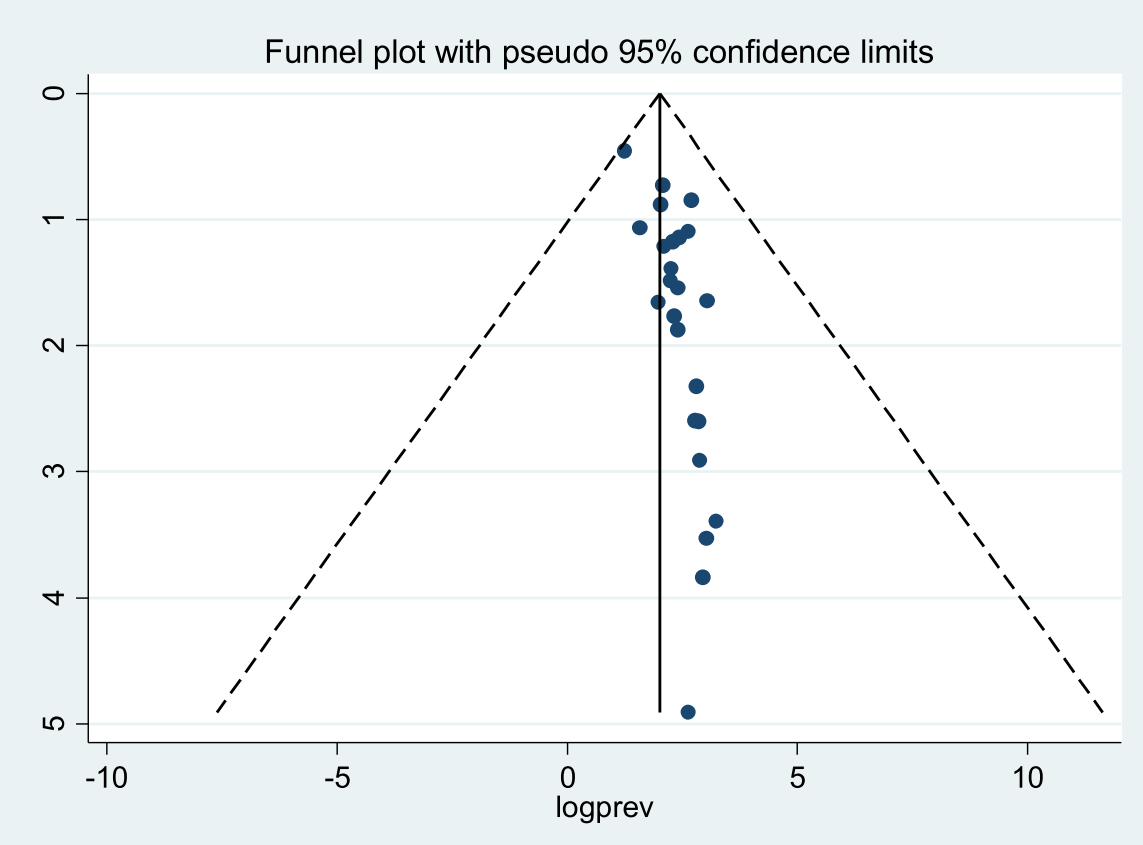

Fig. 3 Funnel plot to test publication bias of the 24 studies 
Filled funnel plot with pseudo $95 \%$ confidence limits

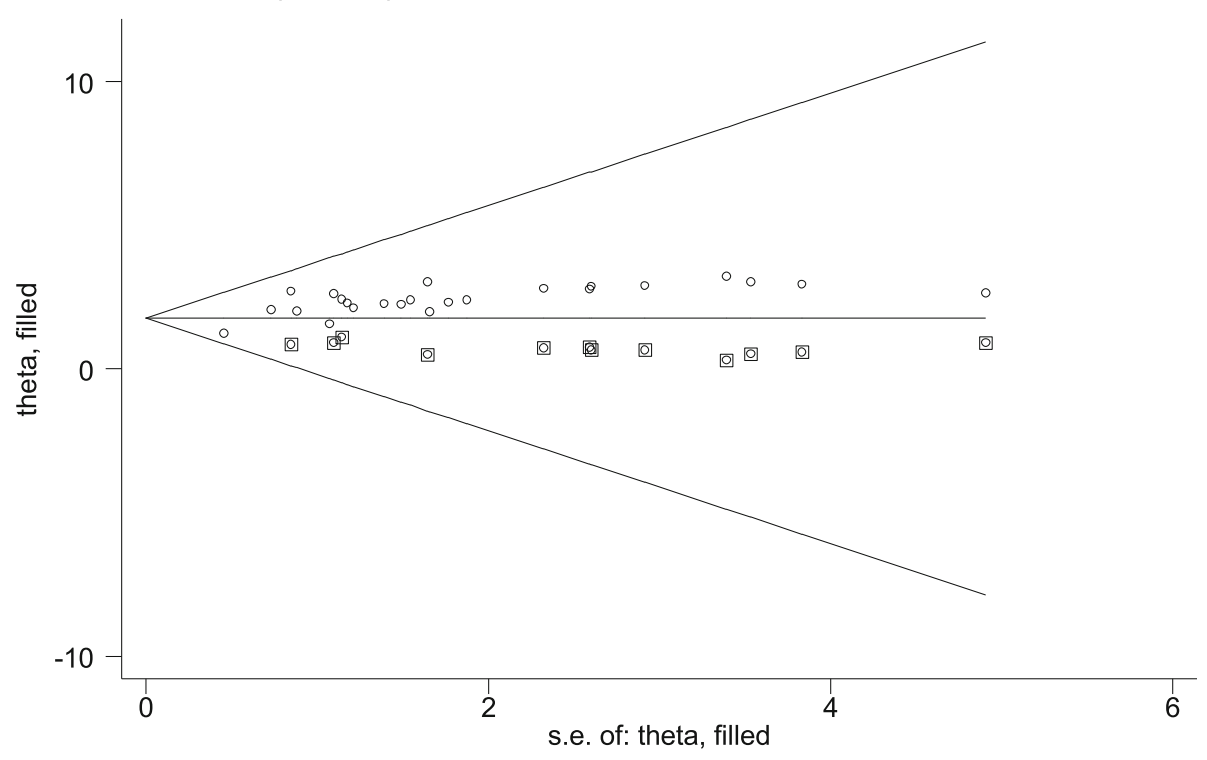

Fig. 4 Result of trim and filled analysis for adjusting publication bias of the 24 studies

\section{Preoperative hospital stay}

The current meta-analysis showed that patients with preoperative hospital stays greater than 7 days were 5.76 times more likely to develop SSIs compared with patients whose preoperative hospital stays were less than or equal to 7 days $\left[\mathrm{AOR}=5.76\right.$; $95 \% \mathrm{CI}: 1.15,28.86, \mathrm{I}^{2}=$ 84.6\%] (Fig. 13). The evidence from Egger's regression test shows that there was no publication bias $(P=0.545)$.

\section{Discussion}

In this meta-analysis the national pooled prevalence of SSIs in Ethiopia was estimated to be $12.3 \%$ (10.19$14.42 \%)$. This finding indicates that SSIs are highly prevalent in hospital patients and reflects inadequate implementation of infection prevention in Ethiopia. Hence, a multifactorial approach is required to manage SSIs, with emphasis being placed on adequate

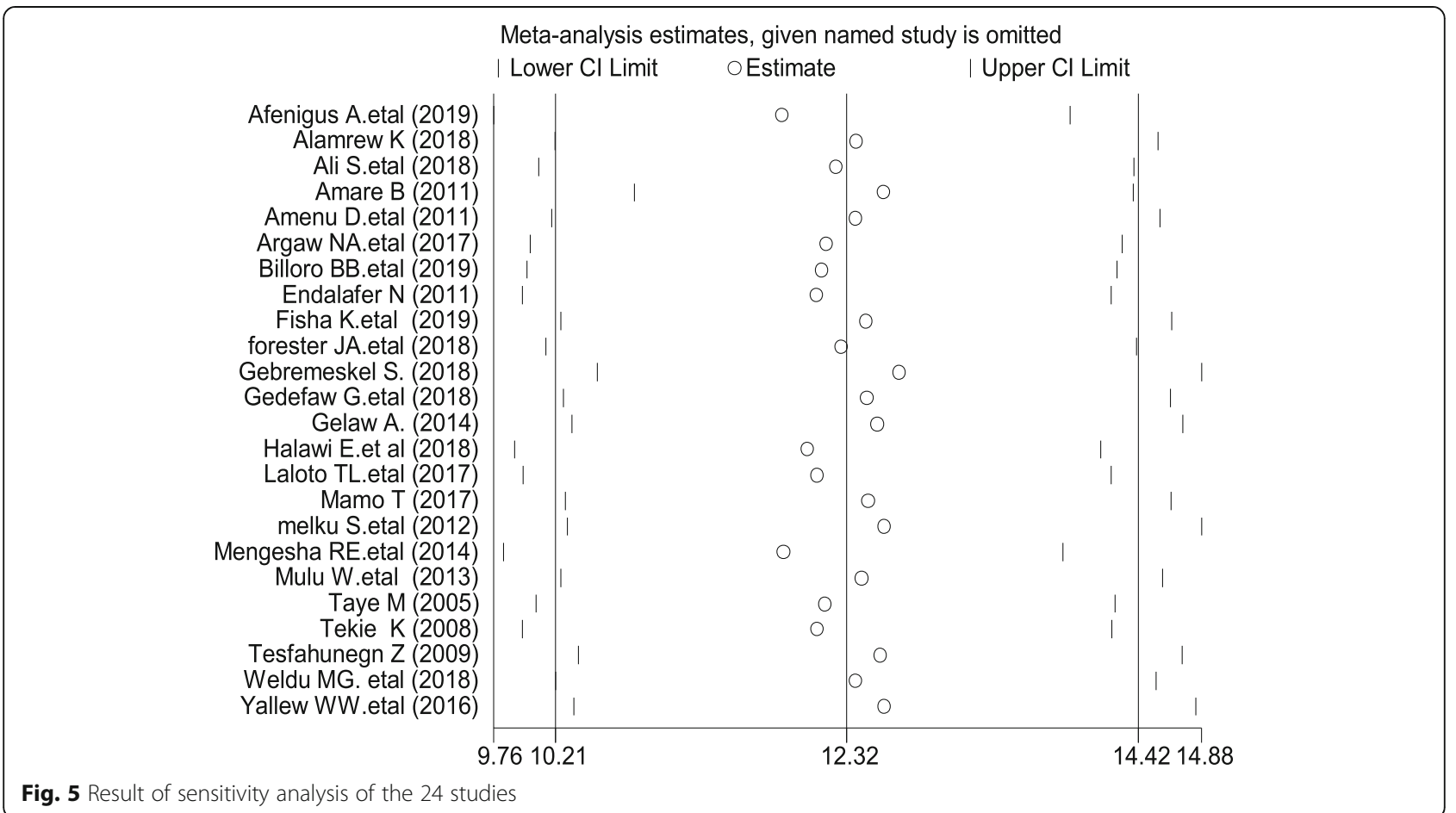




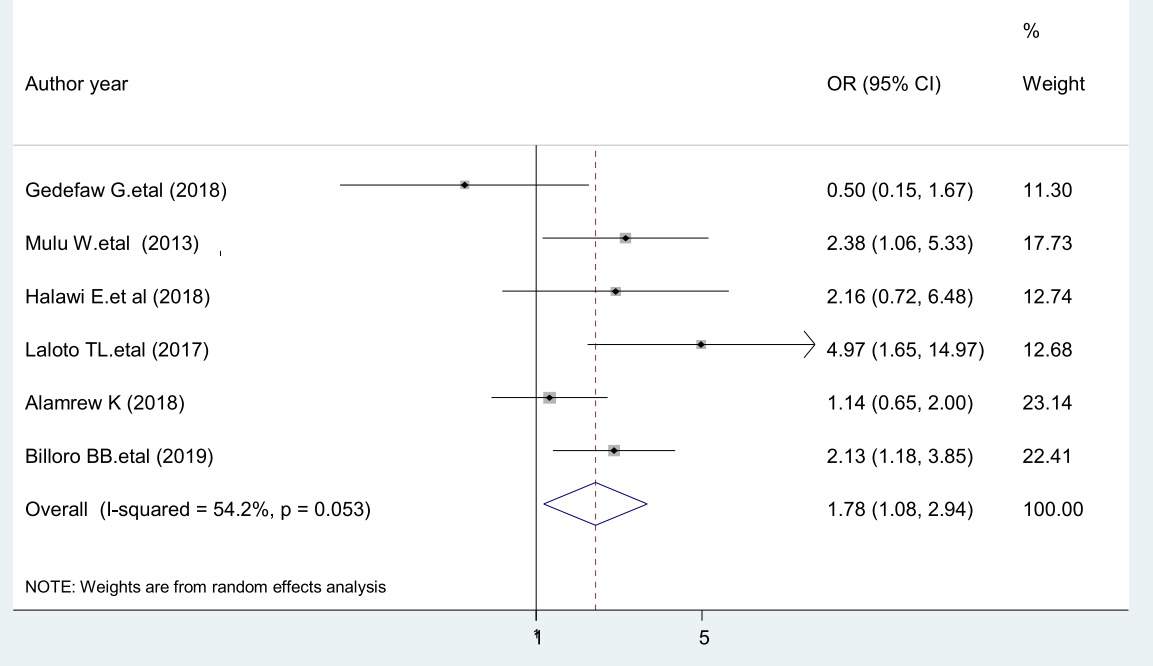

Fig. 6 The pooled effect of duration of operation $>1 \mathrm{~h}$ on surgical site infection

antibiotic prophylaxis, aseptic wound care, and treatment adherence.

Our estimated prevalence of SSIs among postoperative patients in Ethiopia is in line with systematic review and meta-analysis studies done in Nigeria $14.5 \%$ [57] and $14.8 \%$ in Sub-Saharan Africa [58]. However, this result was substantially higher than studies conducted in China was 4.5\% [59] and 5.4\% in Algeria [60]. This variation could be justified by lack of adequate postoperative care, inadequate infection control, lack of up-to-date knowledge, and insufficient trained professionals in Ethiopia. Though the Federal Ministry of Health Ethiopia developed Standard National Guideline on Infection Prevention; study support that healthcare workers had insufficient knowledge and perception on universal precaution [61].

Subgroup analysis in this study showed that the pooled prevalence of SSIs among post-operative patients in SNNPR was 17.19\% (95\% CI: 13.3, 21.09), which was the highest amongst the Ethiopian regions examined, followed by Addis Ababa (14.26\%; 95\% CI: $9.87,18.65)$, whereas the lowest rate was reported in

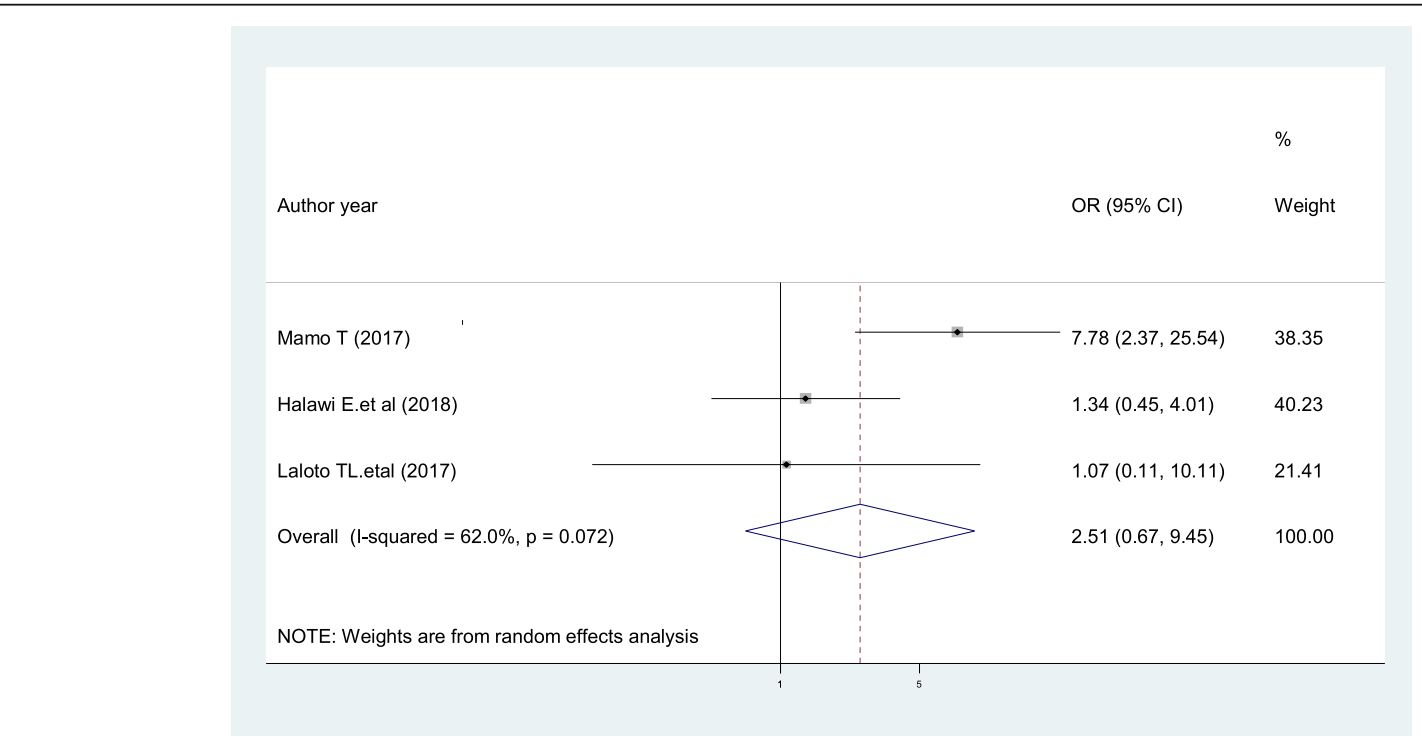

Fig. 7 The pooled effect of preoperative blood transfusion on surgical site infection 


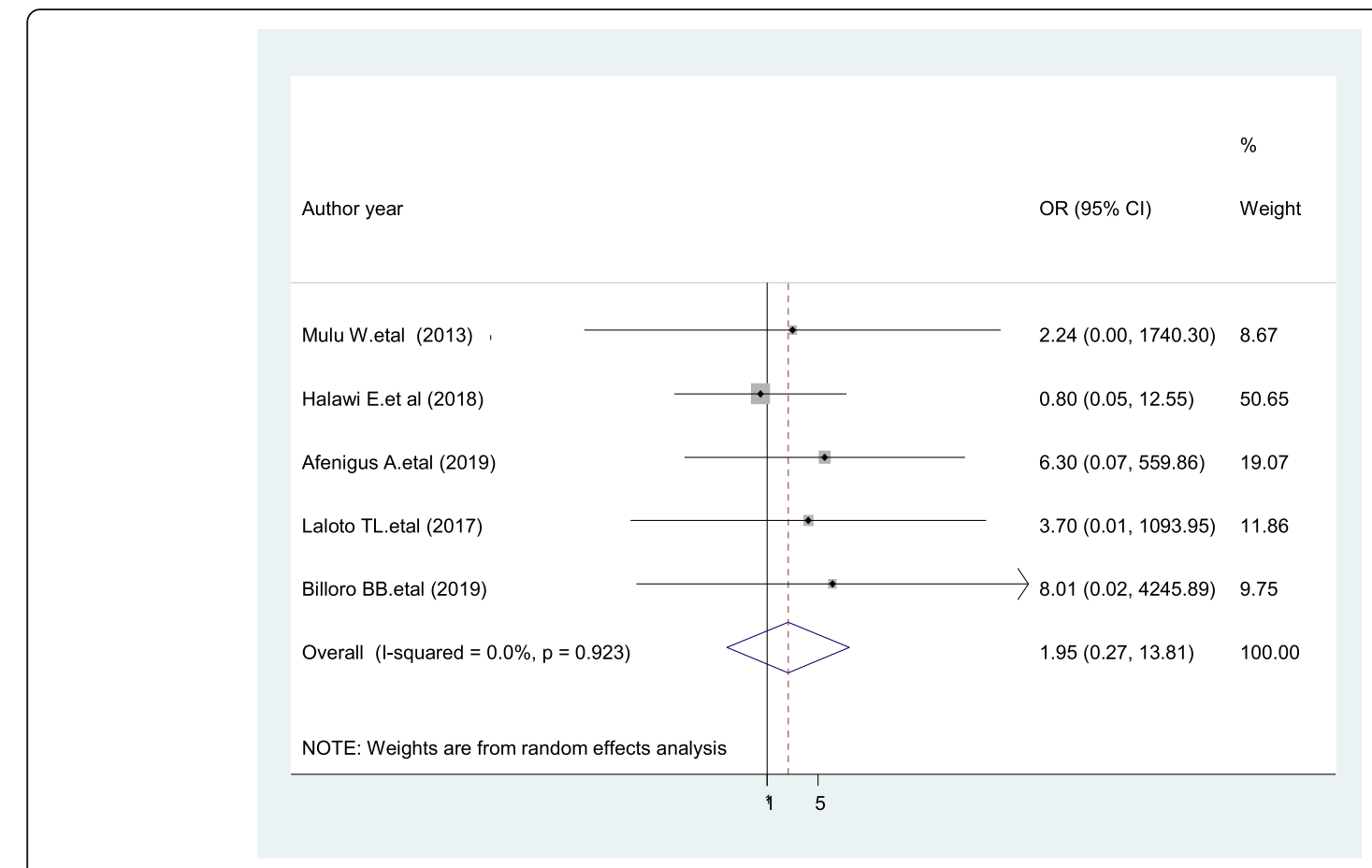

Fig. 8 Forest plot showing the association of smoking and surgical site infection

the Amhara Region (9.36\%; 95\%CI; 6.71, 12.0) of the country. This variation might be due the availability of healthcare resources in the regions, the types of surgery, wound contamination classification, and the diagnostic methods used. Based on the pooled analysis of adjusted odd ratio of studies, duration of operation $>1 \mathrm{~h}$, diabetes mellitus, ASA score $>1$, previous surgery, clean contaminated wound, and preoperative hospital stay $>7$ days were associated with SSIs.

The present study revealed that the duration of operation $>1 \mathrm{~h}$ were nearly two times more at risk to acquire SSIs. This is supported by other studies conducted in Tanzania [17], Nigeria [57], and Spain [62]. Longer duration of surgery would, therefore, increase the risk of surgical wound contamination due to the increased microbial

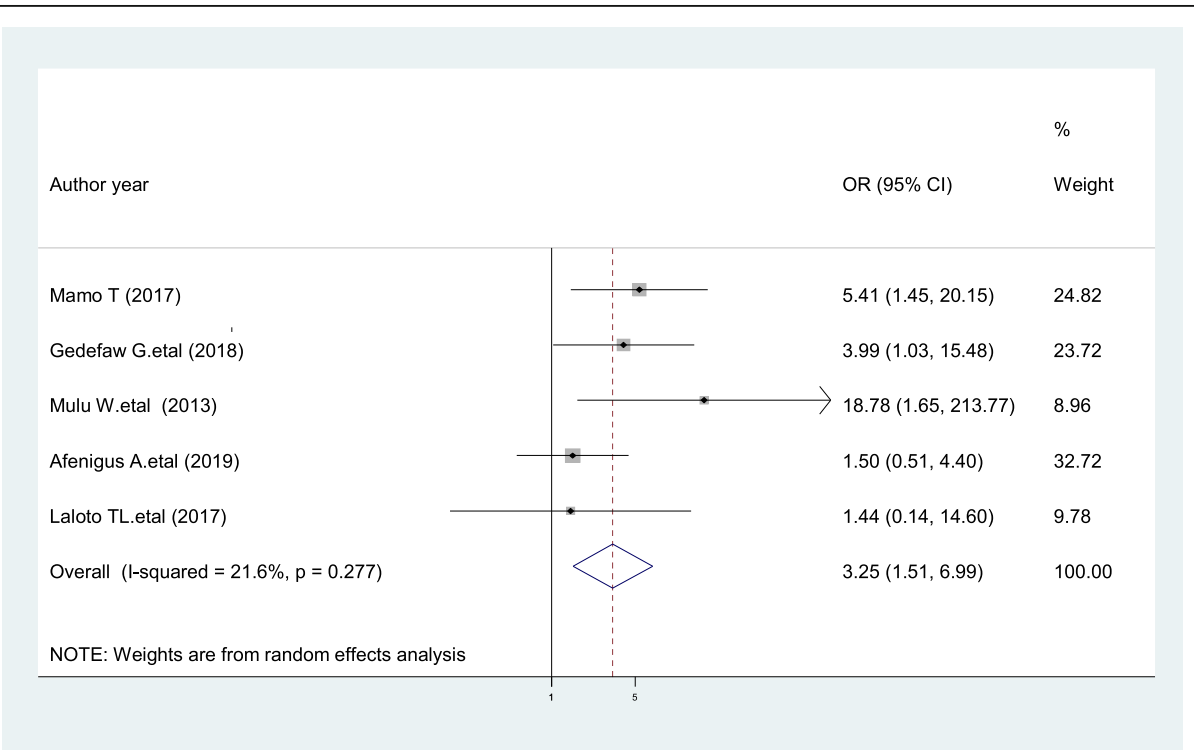

Fig. 9 The pooled effect of diabetes mellitus on surgical site infection 


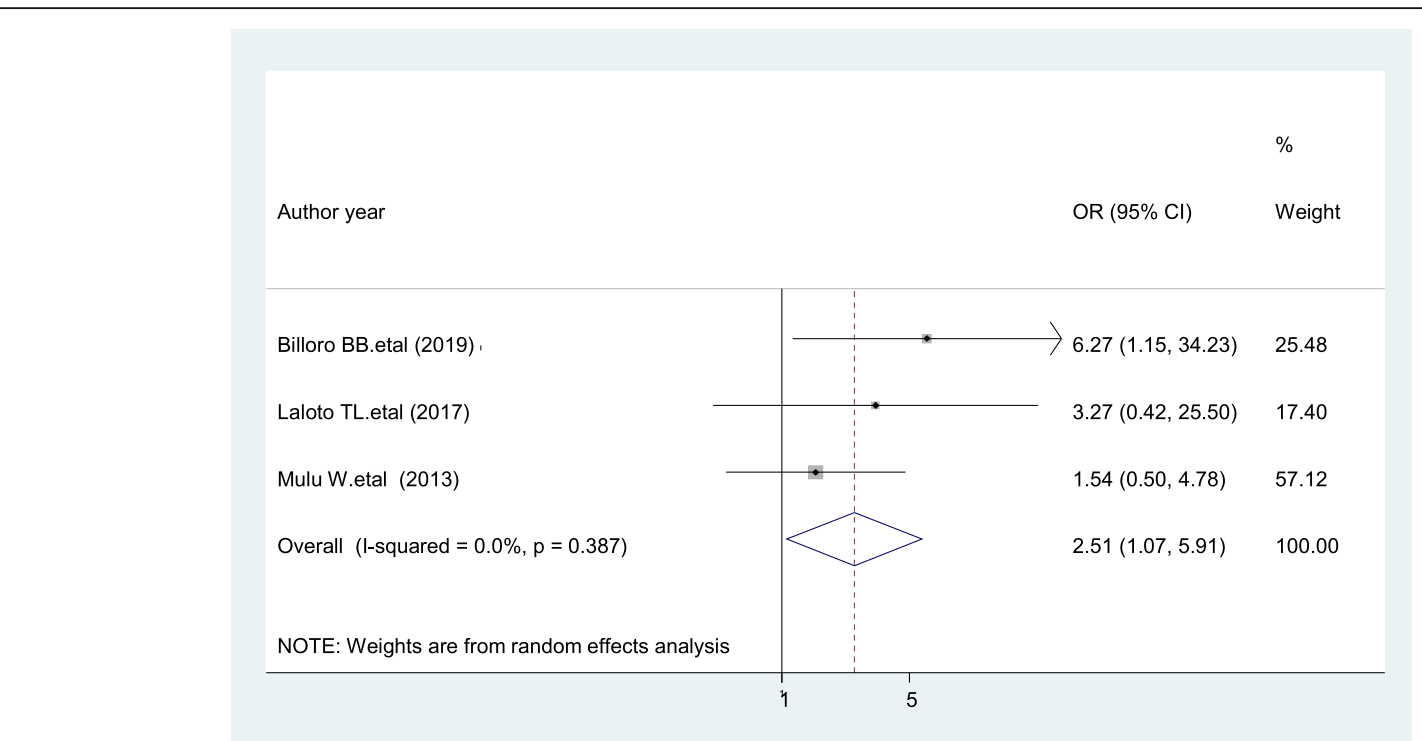

Fig. 10 The pooled effect of ASA score on surgical site infection

exposure in the operation field [63] and it also increases the extent of tissue trauma due to an extensive surgical procedure and increased blood loss which contributes to tissue hypoxia. Increased length of surgical procedure estimated that infection rate nearly doubles with each hour of surgery [64]. Moreover, guidelines recommended limiting the length of the surgery; the longer the incision remains opening, the higher the risk of introduction of microorganisms into the surgical incision [65].

We found that those who had diabetes mellitus were significantly associated with SSIs. This finding supported by other studies conducted in Yemen [66], Nigeria [57], India [67], and Tanzania [17]. Previous studies have shown that patients with pre-existing illnesses, such as diabetes mellitus are at high risk of developing SSI due to their low immunity and that may slow the healing process [68]. Studies have shown that hyperglycemia, particularly from stress, has been associated with a higher risk of SSI. Therefore, evidences recommended that, to optimize the care of patients with diabetes mellitus and reduce the risk of complication, perioperative blood sugar levels should be less than $200 \mathrm{mg} / \mathrm{dl}$ and

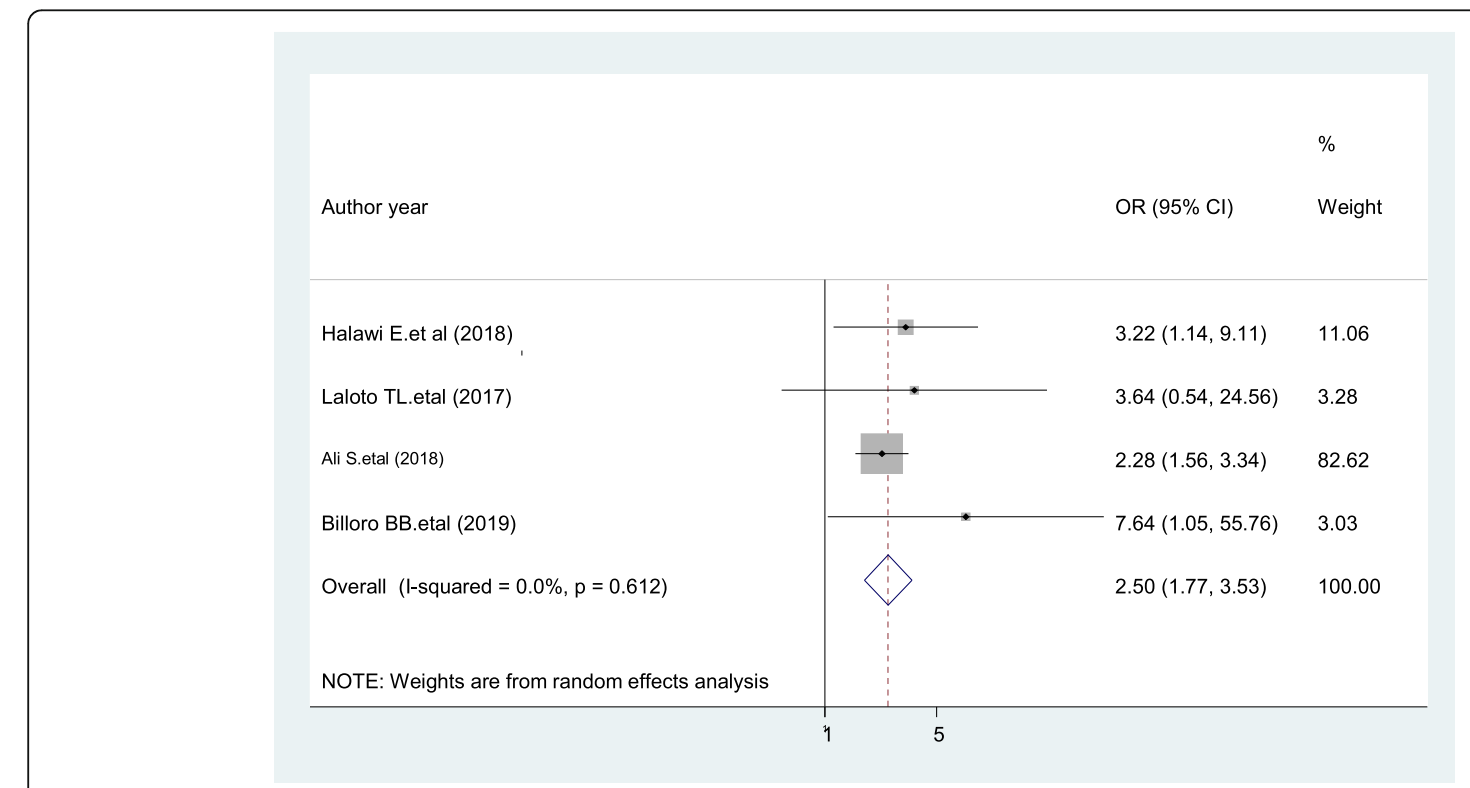

Fig. 11 The pooled effect of previous surgery on surgical site infection 


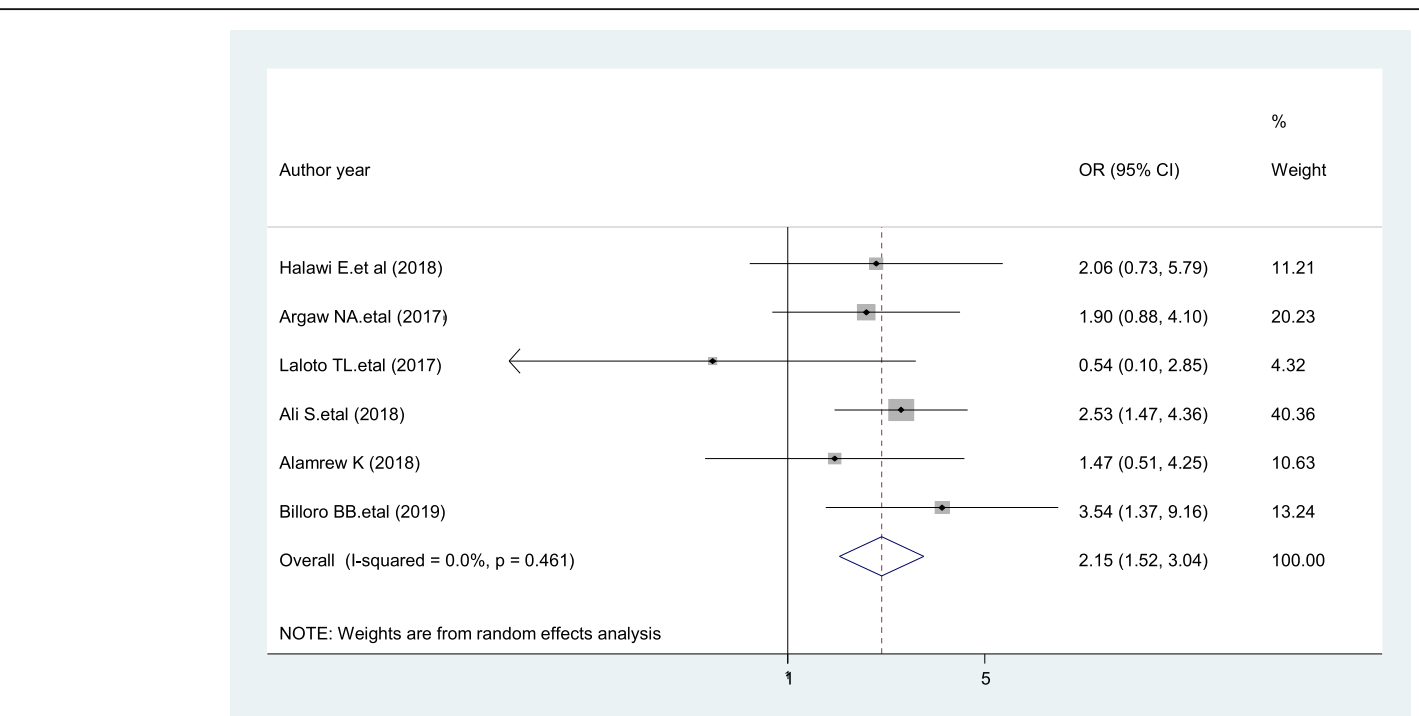

Fig. 12 Forest plot showing the effect of wound type on surgical site infection

HbA1C $<8 \%[65,69,70]$. We found that patients who have ASA score $>1$ were 2.5 times more likely to develop SSIs, which was supported by studies from Canada [71], Uganda [15], Nepal [72], and Cameroon [20]. In addition, global guidelines for the prevention of surgical site infection also supported that an ASA score of at least 3 recognized as factor associated with an increased risk of SSI [73].

Those patients with a history of previous surgery had a significant association with a surgical site infection. This finding supported by other studies conducted in India [67]. The odds of clean contaminated wound were nearly two times to have SSI, which is similar to studies from Cameroon [20], China [59], Nepal [72], and
Uganda [15]. The possible explanation might be previous studies have shown that ceftriaxone is highly sensitive to suspected organisms in clean wound [56], and surgeries with an increased microbial load in the operative field are associated with higher risk of SSI. In addition, evidence support that treating infections on the operating site, if possible, or postponing the surgery until the infection has cleared and if available, consider use of wound protector devices in clean contaminated abdominal surgical procedures in adult patients [65].

In the present review, preoperative hospital stay for more than 7 days increased the risk of SSIs by 5.76 times compared with preoperative hospital stay less than 7 days. In agreement with this finding were studies

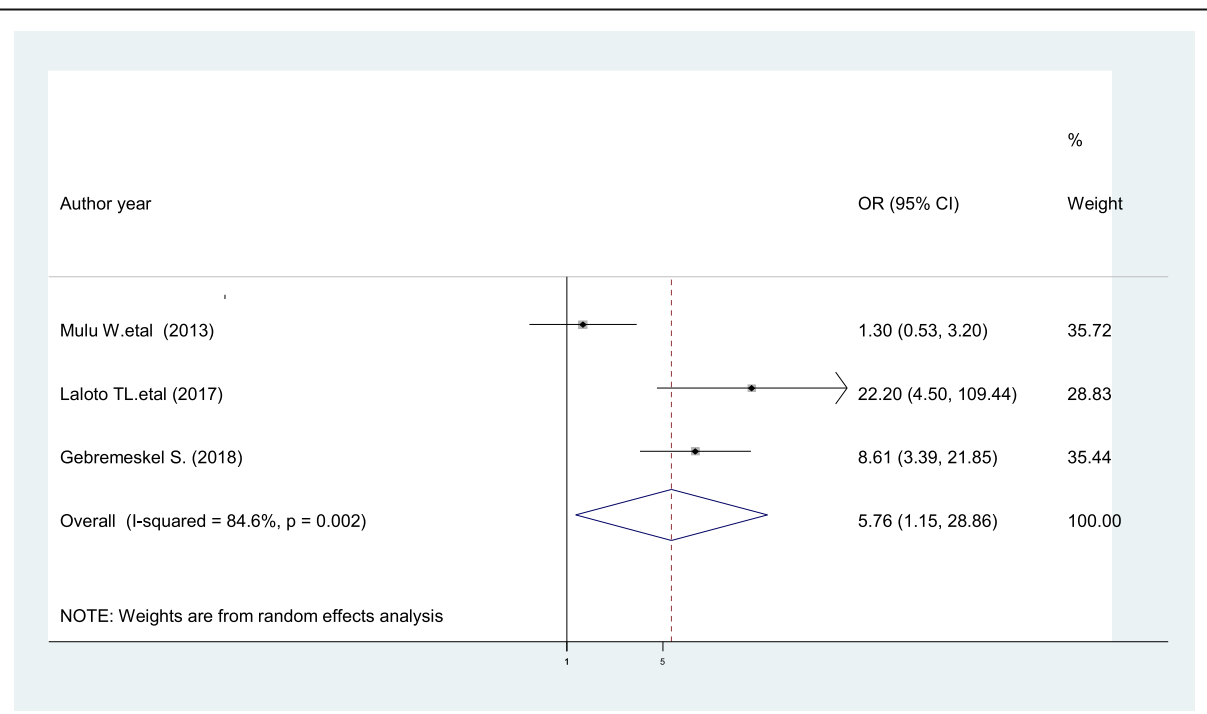

Fig. 13 The pooled effect of preoperative hospital stays on surgical site infection 
conducted in Tanzania [17], and India [67]. In addition, Ethiopia Standard National Guideline on Infection Prevention also supports our findings [64]. The possible explanation might be patients with long stay in hospital before surgery exposes to contamination or colonization by pathogens which will contribute to the occurrence of SSIs [74], suggesting that shortening the preoperative hospital stay reduces the incidence rate of SSIs $[64,73]$. Although preoperative blood transfusions and smoking have been established as risk factors for SSIs in other studies [17, 67], these findings were not supported by our meta-analysis results. Further studies are, therefore, required to validate these observations among Ethiopian patients.

This study has clinical implications in that the high prevalence of SSIs among postoperative patients should guide healthcare professionals to minimize the risk of surgical site infection by providing guidance to the patient who undergone to surgery, give information about possible risk factors during routine patient care, and provide knowledge about wound care. The Nurses' role is considered crucial in optimizing the healing outcomes through aseptic wound care; provision of explicit patient instructions on how to care for their wound, education and counseling and understanding of the patient's needs. In addition, identifying associated risk factors may help health care professionals treat SSIs patients during their clinical care.

The current meta-analysis has limitations that should be considered in future research. First, it may be lacked national representativeness because no data were found from all region of Ethiopia. Second, it was challenging to synthesise some of the factors as they were not defined or measured in the same way across the different studies. Hence, further study is required to identify associated factors for the development of SSI among postoperative patients.

\section{Conclusions}

This study revealed that the prevalence of SSIs remains high among postoperative patients in Ethiopia based on the 24 research-based papers included in this study. That said, the prevalence of SSIs differed by region. Therefore, situation-based interventions and region context-specific preventive strategies should be developed to reduce the prevalence of SSI. A more comprehensive consideration of the existing evidence will potentially inform effective strategies for preventing SSI within the Ethiopian context. In addition, this meta-analysis may help policy-makers and program managers to design interventions on preventing the occurrence of SSIs.

\section{Abbreviations}

ASA: American Society of Anaesthesiologists; Cl: Confidence Interval, AOR: Adjusted Odds Ratio; PRISMA: Preferred Reporting Items for Systematic Reviews and Meta-Analyses; SNNPR: Southern Nations Nationalities and Peoples Region; SSI: Surgical Site Infection.

\section{Acknowledgements}

Not applicable.

\section{Authors' contributions}

WSS and TYA developed the protocol and were involved in the design, selection of study, data extraction, statistical analysis, and developing the initial drafts of the manuscript. YAA, TYA and PMP were involved in data extraction, quality assessment, statistical analysis and revising. WSS, YAA, and PMP prepared and edited the final draft of the manuscript. All authors read and approved the final draft of the manuscript.

\section{Funding}

Not applicable.

\section{Availability of data and materials}

The data analysed during the current meta-analysis is available from the corresponding author on reasonable request.

Ethics approval and consent to participate

Not applicable.

\section{Consent for publication}

Not applicable.

\section{Competing interests}

The authors declare that they have no competing interests.

\section{Author details}

${ }^{1}$ Department of Nursing, College of Health Science, Debre Berhan University, P.O. Box 445, Debre Berhan, Ethiopia. ${ }^{2}$ Department of Nursing, College of Health Science, Debre Markos University, Debre Markos, Ethiopia. ${ }^{3}$ College of Nursing, University of Saskatchewan, Saskatoon, Canada.

Received: 3 January 2020 Accepted: 30 April 2020

Published online: 18 May 2020

\section{References}

1. Smith MA, Dahlen NR, Bruemmer A, Davis S, Heishman C. Clinical practice guideline surgical site infection prevention. Orthop Nurs. 2013;32(5):242-8.

2. Allegranzi B, Nejad SB, Combescure C, Graafmans W, Attar $H_{\text {, }}$ Donaldson L, Pittet D. Burden of endemic health-care-associated infection in developing countries: systematic review and meta-analysis. Lancet. 2011;377(9761):228-41

3. Organization $\mathrm{WH}$ : The burden of health care-associated infection worldwide: A summary. In.; 2010.

4. Amoran O, Sogebi A, Fatugase $\mathrm{O}$. Rates and risk factors associated with surgical site infections in a tertiary Care Center in South-Western Nigeria; 2013

5. Organization WH: Preventing surgical site infections: implementation approaches for evidence-based recommendations. 2018.

6. Curcio D, Cane A, Fernández F, Correa J. Surgical site infection in elective clean and clean-contaminated surgeries in developing countries. Int J Infect Dis. 2019:80:34-45.

7. Palumbo VD, Bruno A, Di Trapani B, Tomasello G. Who global guidelines for the prevention of surgical site infection: a new step to improve PATIENTS' SAFETY before, DURING AND AFTER SURGERY. 2016;2017.

8. Smyth E, Mcllvenny G, Enstone J, Emmerson A, Humphreys H, Fitzpatrick F, Davies E, Newcombe R, Spencer R, Group HISPSS. Four country healthcare associated infection prevalence survey 2006: overview of the results. J Hosp Infect. 2008;69(3):230-48

9. Danzmann L, Gastmeier P, Schwab F, Vonberg R-P. Health care workers causing large nosocomial outbreaks: a systematic review. BMC Infect Dis. 2013;13(1):98

10. Ott E, Saathoff S, Graf K, Schwab F, Chaberny IF. The prevalence of nosocomial and community acquired infections in a university hospital: an observational study. Dtsch Arztebl Int. 2013;110(31-32):533.

11. Organization $W H$ : Global guidelines for the prevention of surgical site infection. 2016. World Health Organization website http://www.who.int/ gpsc/ssi-prevention-guidelines/en 2018. 
12. Nejad SB, Allegranzi B, Syed SB, Ellis B, Pittet D. Health-care-associated infection in Africa: a systematic review. Bull World Health Organ. 2011;89: 757-65.

13. NWOSE PC. A prospective study of the incidence of surgical wound infection at the NNAMDI AZIKIWE UNIVERSITY teaching hospital. Faculty of SURGERY: NNEWl; 2006.

14. Nobandegani Zinat M, Doulatabad Shahla N, Masoumeh R, Ardeshir A. Surgical site infection incidence after a clean-contaminated surgery in Yasuj Shahid Beheshti hospital. Iran Investigación y Educación en Enfermería. 2011:29(3):435-41.

15. Lubega A, Joel B, Justina Lucy N. Incidence and etiology of surgical site infections among emergency postoperative patients in Mbarara regional referral hospital. South Western Uganda Surgery research and practice. 2017;2017.

16. Mulu W, Kibru G, Beyene G, Damtie H. Associated risk factors for postoperative nosocomial infections among patients admitted at Felege Hiwot referral hospital, Bahir Dar. Northwest Ethiopia Clin Med Res. 2013; 2(6):140-7.

17. Mawalla B, Mshana SE, Chalya PL, Imirzalioglu C, Mahalu W. Predictors of surgical site infections among patients undergoing major surgery at Bugando medical Centre in Northwestern Tanzania. BMC Surg. 2011; 11(1):21.

18. Afenigus A, Shbabawu A, Melese T. Surgical site infection and associated factors among adult patients admitted in west and east Gojjam zone hospitals, Amhara region. Ethiopia Nurse Care Open Acces J. 2019;6(3):107-12.

19. Weldu MG, Berhane H, Berhe N, Haile K, Sibhatu Y, Gidey T, Amare K, Zelalem H, Mezemir R, Hadgu T. Magnitude and determinant factors of surgical site infection in Suhul hospital Tigrai, northern Ethiopia: a crosssectional study. Surg Infect. 2018;19(7):684-90

20. Yaouba D, Ngah JE, Perpoint T, Mbo AJ, Vanhems P, Bénet T. Incidence and risk factors for surgical site infections in N'Gaoundéré regional hospital, Cameroon. Am J Infect Control. 2016;44(10):1195.

21. Laloto TL, Gemeda DH, Abdella SH. Incidence and predictors of surgical site infection in Ethiopia: prospective cohort. BMC Infect Dis. 2017;17(1):119.

22. Hartmann $M$, Jönsson $K$, Zederfeldt B: Effect of tissue perfusion and oxygenation on accumulation of collagen in healing wounds. Randomized study in patients after major abdominal operations. The European journal of surgery= Acta chirurgica 1992, 158(10):521-526.

23. Halawi E, Assefa T, Hussen S. Pattern of antibiotics use, incidence and predictors of surgical site infections in a tertiary care teaching hospital. BMC research notes. 2018;11(1):538.

24. de Lissovoy G, Fraeman K, Hutchins V, Murphy D, Song D, Vaughn BB. Surgical site infection: incidence and impact on hospital utilization and treatment costs. Am J Infect Control. 2009;37(5):387-97.

25. Kitembo S, Chugulu S: Incidence of surgical site infections and microbial pattern at kilimanjaro christian medical centre. Annals of African Surgery 2013, 10(1).

26. Badia JM, Casey A, Petrosillo N, Hudson P, Mitchell S, Crosby C. Impact of surgical site infection on healthcare costs and patient outcomes: a systematic review in six European countries. J Hosp Infect. 2017;96(1):1-15.

27. Coello R, Charlett A, Wilson J, Ward V, Pearson A, Borriello P. Adverse impact of surgical site infections in English hospitals. J Hosp Infect. 2005;60(2):93103.

28. Jenks P, Laurent M, McQuarry S, Watkins R. Clinical and economic burden of surgical site infection (SSI) and predicted financial consequences of elimination of SSI from an English hospital. J Hosp Infect. 2014;86(1):24-33.

29. Kanellakopoulou K, Papadopoulos A, Varvaroussis D, Varvaroussis A, Giamarellos-Bourboulis EJ, Pagonas A, Stergiou A, Papadelis P, Nikolaidis V, Giamarellou $\mathrm{H}$. Efficacy of teicoplanin for the prevention of surgical site infections after total hip or knee arthroplasty: a prospective, open-label study. Int J Antimicrob Agents. 2009;33(5):437-40.

30. Allegranzi B, Aiken AM, Kubilay NZ, Nthumba P, Barasa J, Okumu G, Mugarura R, Elobu A, Jombwe J, Maimbo M. A multimodal infection control and patient safety intervention to reduce surgical site infections in Africa: a multicentre, before-after, cohort study. Lancet Infect Dis. 2018;18(5):507-15.

31. Peters MD, Godfrey CM, Mclnerney P, Soares CB, Khalil H, Parker D. The joanna briggs institute reviewers' manual 2015: methodology for JB scoping reviews; 2015.

32. DerSimonian R, Laird N. Meta-analysis in clinical trials. Control Clin Trials. 1986;7(3):177-88.
33. Huedo-Medina TB, Sánchez-Meca J, Marín-Martínez F, Botella J. Assessing heterogeneity in meta-analysis: Q statistic or $\mathrm{I}^{2}$ index? Psychol Methods. 2006;11(2):193.

34. Higgins JP, Altman DG. Assessing risk of bias in included studies. Cochrane handbook for systematic reviews of interventions: Cochrane book series. 2008:187-241.

35. Egger M, Davey-Smith G, Altman D. Systematic reviews in health care: metaanalysis in context: John Wiley \& Sons; 2008.

36. StataCorp L. Stata statistical software (version release 14). Author: College Station, TX; 2015.

37. Moher D, Liberati A, Tetzlaff J, Altman DG. Preferred reporting items for systematic reviews and meta-analyses: the PRISMA statement. Ann Intern Med. 2009:151(4):264-9.

38. Forrester JA, Koritsanszky L, Parsons BD, Hailu M, Amenu D, Alemu S, Jiru F, Weiser TG. Development of a surgical infection surveillance program at a tertiary hospital in Ethiopia: lessons learned from two surveillance strategies. Surg Infect. 2018;19(1):25-32.

39. Amare B, Abdurrahman Z, Moges B, Ali J, Muluken L, Alemayehu M, Yifru S, Sendek B, Belyhun Y, Moges F. Postoperative surgical site bacterial infections and drug susceptibility patterns at Gondar University teaching hospital. Northwest Ethiopia J Bacteriol Parasitol. 2011;2(8):126.

40. Alamrew K. Assessment of antibiotics use as surgical prophylaxis and postoperative treatment at Saint Paul's hospital millennium medical college: Addis Ababa Universty; 2018.

41. Argaw NA, Shumbash KZ, Asfaw AA, Hawaze S. Assessment of surgical antimicrobial prophylaxis in orthopaedics and traumatology surgical unit of a tertiary care teaching Hospital in Addis Ababa. BMC research notes. 2017; 10(1):160.

42. Endalafer N. Bacterial nosocomial infections and their antimicrobial susceptibility patterns in surgical wards and surgical intensive care unit (SICU) of Tikur Anbessa University hospital Addis Ababa. Ethiopia: Addis Ababa University; 2008.

43. Gebremeskel S. Management of Hospital-Acquired Infections among hospitalized patients at Zewditu memorial hospital. Addis Ababa, Ethiopia: A Prospective Study. Addis Ababa Universty; 2018.

44. Taye M. Wound infection in Tikur Anbessa hospital, surgical department. Ethiop Med J. 2005;43(3):167-74.

45. Tekie K: Surgical wound infection in Tikur Anbessa hospital with special emphasis on Pseudomonas aeruginosa. Unpublished MSc thesis in medical microbiology, Addis Ababa University, Medical Faculty, Ethiopia http://etd. aau.edu.et/bitstream/handle/123456789/5772/Kassaye\%20Tekie.pdf (Accessed on January 31, 2014) 2008.

46. Fisha K, Azage M, Mulat G, Tamirat KS. The prevalence and root causes of surgical site infections in public versus private hospitals in Ethiopia: a retrospective observational cohort study. Patient safety in surgery. 2019; 13(1):26.

47. Gedefaw G, Asires A, Shiferaw S, Addisu D. Factors associated with surgical site infection among women undergoing obstetrics surgery at Felegehiwot referral hospital, Bahir Dar, Northwest Ethiopia: a retrospective crosssectional study. Safety in Health. 2018;4(1):14.

48. Gelaw A, Gebre-Selassie S, Tiruneh M, Mathios E, Yifru S. Isolation of bacterial pathogens from patients with postoperative surgical site infections and possible sources of infections at the University of Gondar Hospital. Northwest Ethiopia J Environ Occup Sci. 2014;3(2):103-8.

49. Melaku S, Gebre-Selassie S, Damtie M, Alamrew K. Hospital acquired infections among surgical, gynaecology and obstetrics patients in FelegeHiwot referral hospital, Bahir Dar, Northwest Ethiopia. Ethiop Med J. 2012; 50(2):135-44.

50. Yallew WW, Kumie A, Yehuala FM: Point prevalence of hospital-acquired infections in two teaching hospitals of Amhara region in Ethiopia. Drug, healthcare and patient safety 2016, 8:71.

51. Ali S, Birhane M, Bekele S, Kibru G, Teshager L, Yilma Y, Ahmed Y, Fentahun $\mathrm{N}$, Assefa $\mathrm{H}$, Gashaw M. Healthcare associated infection and its risk factors among patients admitted to a tertiary hospital in Ethiopia: Iongitudinal study. Antimicrobial Resistance \& Infection Control. 2018;7(1):2.

52. Amenu D, Belachew T, Araya F. Surgical site infection rate and risk factors among obstetric cases of Jimma University specialized hospital, Southwest Ethiopia. Ethiop J Health Sci. 2011;21(2):91-100.

53. Mamo T, Abebe TW, Chichiabellu TY, Anjulo AA. Risk factors for surgical site infections in obstetrics: a retrospective study in an Ethiopian referral hospital. Patient safety in surgery. 2017;11(1):24. 
54. Mengesha RE, Kasa BG-S, Saravanan M, Berhe DF, Wasihun AG. Aerobic bacteria in post surgical wound infections and pattern of their antimicrobial susceptibility in Ayder teaching and referral hospital, Mekelle, Ethiopia. BMC research notes. 2014;7(1):575.

55. Tesfahunegn Z, Asrat D, Woldeamanuel Y, Estifanos K. Bacteriology of surgical site and catheter related urinary tract infections among patients admitted in Mekelle hospital, Mekelle, Tigray, Ethiopia. Ethiop Med J. 2009;47(2):117-27.

56. Billoro BB, Nunemo MH, Gelan SE. Evaluation of antimicrobial prophylaxis use and rate of surgical site infection in surgical ward of Wachemo University Nigist Eleni Mohammed memorial hospital, southern Ethiopia: prospective cohort study. BMC Infect Dis. 2019;19(1):298.

57. Olowo-Okere A, Ibrahim YKE, Olayinka BO, Ehinmidu JO. Epidemiology of surgical site infections in Nigeria: a systematic review and meta-analysis. Nigerian Postgraduate Medical Journal. 2019;26(3):143.

58. Ngah J, Bénet T, Djibrilla Y. Incidence of surgical site infections in subSaharan Africa: systematic review and meta-analysis. In: African Field Epidemiology Network. 2016.

59. Fan $Y$, Wei Z, Wang W, Tan L, Jiang H, Tian L, Cao Y, Nie S. The incidence and distribution of surgical site infection in mainland China: a meta-analysis of 84 prospective observational studies. Sci Rep. 2014;4:6783.

60. Atif M, Azouaou A, Bouadda N, Bezzaoucha A, Si-Ahmed M, Bellouni R. Incidence and predictors of surgical site infection in a general surgery department in Algeria. Revue d'epidemiologie et de sante publique. 2015;63(4):275-9.

61. Reda AA, Fisseha S, Mengistie B, Vandeweerd J-M: Standard precautions: occupational exposure and behavior of health care workers in Ethiopia. PLoS One 2010, 5(12).

62. Hijas-Gómez Al, Lucas WC, Checa-García A, Martínez-Martín J, FahandezhSaddi H, Gil-de-Miguel Á, Durán-Poveda M, Rodríguez-Caravaca G. Surgical site infection incidence and risk factors in knee arthroplasty: a 9-year prospective cohort study at a university teaching hospital in Spain. Am J Infect Control. 2018;46(12):1335-40.

63. Thanni LO, Aigoro NO. Surgical site infection complicating internal fixation of fractures: incidence and risk factors. J Natl Med Assoc. 2004;96(8):1070.

64. Federal minister of Ethiopia. Infection prevention and patient's safety training resource packageparticipant'smanual.https://aidsfree.usaid.gov/sites/ default/files/ipps_participant_manualweb.pdf. Accessed March 10, 2020.

65. Curless M, Ruparelia C, Thompson E, Trexler P. Infection prevention and control: reference manual for health care facilities with limited resources. Baltimore, MD, USA: Jhpiego; 2018.

66. Nasser A, Zhang X, Yang L, Sawafta FJ, Salah B. Assessment of surgical site infections from signs \& symptoms of the wound and associated factors in public hospitals of Hodeidah City. Yemen Int J Appl. 2013;3(3):101-10.

67. Setty NKH, Nagaraja MS, Nagappa DH, Giriyaiah CS, Gowda NR, Naik RDML: A study on Surgical Site Infections (SSI) and associated factors in a government tertiary care teaching hospital in Mysore, Karnataka. International Journal of Medicine and Public Health 2014, 4(2).

68. Delamaire M, Maugendre D, Moreno M, Le Goff MC, Allannic H, Genetet B. Impaired leucocyte functions in diabetic patients. Diabet Med. 1997;14(1):29-34.

69. Ling ML, Apisarnthanarak A, Abbas A, Morikane K, Lee KY, Warrier A, Yamada K. APSIC guidelines for the prevention of surgical site infections. Antimicrobial Resistance \& Infection Control. 2019;8(1):1-8.

70. Barbara M. Soule. Evidence-Based Principles and Practices for Preventing Surgical Site Infections.https://www.jointcommissioninternational.org/-/ media/jci/jci-documents/offerings/advisory-services/infection-preventionand-control/jci_ssi_toolkit.pdf?db=web\&hash=51FCBF18BC050870C6721734 A67F79ED Accessed April 1, 2020

71. Chattopadhyay R, Zaroukian S, Potvin E. Surgical site infection rates at the Pontiac health care Centre, a rural community hospital. Can J Rural Med. 2006;11(1):41-8

72. Shrestha S, Wenju P, Shrestha R, Karmacharya R. Incidence and risk factors of surgical site infections in Kathmandu university hospital, Kavre. Nepal Kathmandu University Medical Journal. 2016;14(54):107-11.

73. Leaper D, Edmiston C. World Health Organization: global guidelines for the prevention of surgical site infection. J Hosp Infect. 2017;95(2):135-6.

74. Banashankari $\mathrm{G}$, Rudresh $\mathrm{H}$, Harsha S: An overview of surgical site infections: an insight into the prevalence, etiology and predisposing factors. Valley Int J 204AD, 1(9):493-499.

\section{Publisher's Note}

Springer Nature remains neutral with regard to jurisdictional claims in published maps and institutional affiliations.

\section{Ready to submit your research? Choose BMC and benefit from:}

- fast, convenient online submission

- thorough peer review by experienced researchers in your field

- rapid publication on acceptance

- support for research data, including large and complex data types

- gold Open Access which fosters wider collaboration and increased citations

- maximum visibility for your research: over $100 \mathrm{M}$ website views per year

At BMC, research is always in progress.

Learn more biomedcentral.com/submissions 